\title{
Identifying speeds and accelerations for the systems within a didactic stand operated by means of stepper motors
}

\author{
Emilia-Maria Popescu ${ }^{1}$, Constantin-Adrian Popescu ${ }^{1}$, Cicerone Laurentiu Popa ${ }^{1,{ }^{*}}$, Gabriel- \\ Tiberiu Dobrescu ${ }^{1}$ and Costel Emil Cotet $^{1}$ \\ ${ }^{1}$ University Politehnica of Bucharest, RSP Department, Spl. Independentei 313, Bucharest, Romania
}

\begin{abstract}
The present paper aims to highlight the way in which the speeds and accelerations for the numerical control (NC) axes of the systems specific to a didactic stand with Automated Storage \& Retrieval System (AS-RS) and guided transport system can be determined experimentally. The stand consists of an AS-RS system where the storage of pallets with products is achieved using 3 storage structures arranged circularly around the transport-transfer system. The pallets reach the entryexit station by means of a two-station guided transport system. Its role is to supply the AS-RS with pallets for storage and to evacuate the pallets from it. Stepper motors were used to drive the $\mathrm{NC}$ axes specific to the mentioned systems, and the control was realized by means of programmable logical controllers (PLC). The control programs were made in applications according to these PLC. A Supervisory control and data acquisition (SCADA) type interface was created and thus it was possible to determine experimentally the speeds and accelerations specific to the $\mathrm{NC}$ axes of the mentioned systems.
\end{abstract}

\section{Introduction}

Stepper motors are used in many applications where position control and the ability to maintain position are required, such as: numerically controlled machines (CNC), engraving equipment, robots and handling systems, 3D printers, etc. [1,2].

Numerous studies have been done over the years on the speeds and accelerations of stepper motors.

For the general case, when the speed changes in the acceleration and deceleration phases are different, the speed profile specific to stepper motors can be achieved in real time, by means of speed control algorithms. In order to control the speed of stepper motors within the specified systems, the values of accelerations and decelerations must be defined in advance [3]. The influence of acceleration and deceleration curves on the performance of stepper motors can be analyzed by optimizing the open loop control system of the motor, based on its operating principles [4].

\footnotetext{
Corresponding author: laur.popa79@gmail.com
} 
The creation of an educational stand with $\mathrm{NC}$ axes driven by stepper motors allowed their testing and study for different operating regimes. The studies led to the optimization of the performances of the systems within the educational stand, by the variation of the functional parameters of the stepper motors from the structure of the $\mathrm{CN}$ axes. Thus, experimental data could also be used to simulate material flow for different operating scenarios [5].

\section{Didactic stand used for experimental research}

The experimental research was carried out on a teaching stand that has in its component an automatic storage system (AS-RS), a two-station robocar that has the role of ensuring the supply and retrieval of pallets from the entry-exit area of AS-RS and an area with 3 intermediate storage stations (Figure 1).

The AS-RS specific transport-transfer system has $3 \mathrm{CN}$ axes, a rotation at the base and a vertical translation for positioning near the specific storage location and a horizontal translation for taking over or depositing the pallets.

The robocar has in its component $3 \mathrm{CN}$ axes, a $\mathrm{CN}$ axis ensures its movement on the ground between the intermediate storage stations and the specific input-output area AS-RS and the other two $\mathrm{CN}$ axes ensure the taking over and deposition of the pallets.

The $6 \mathrm{NC}$ axes are driven by stepper motors, and the movement is transmitted by various mechanisms (pinion-rack, belt-belt wheels, driver-nut screw).

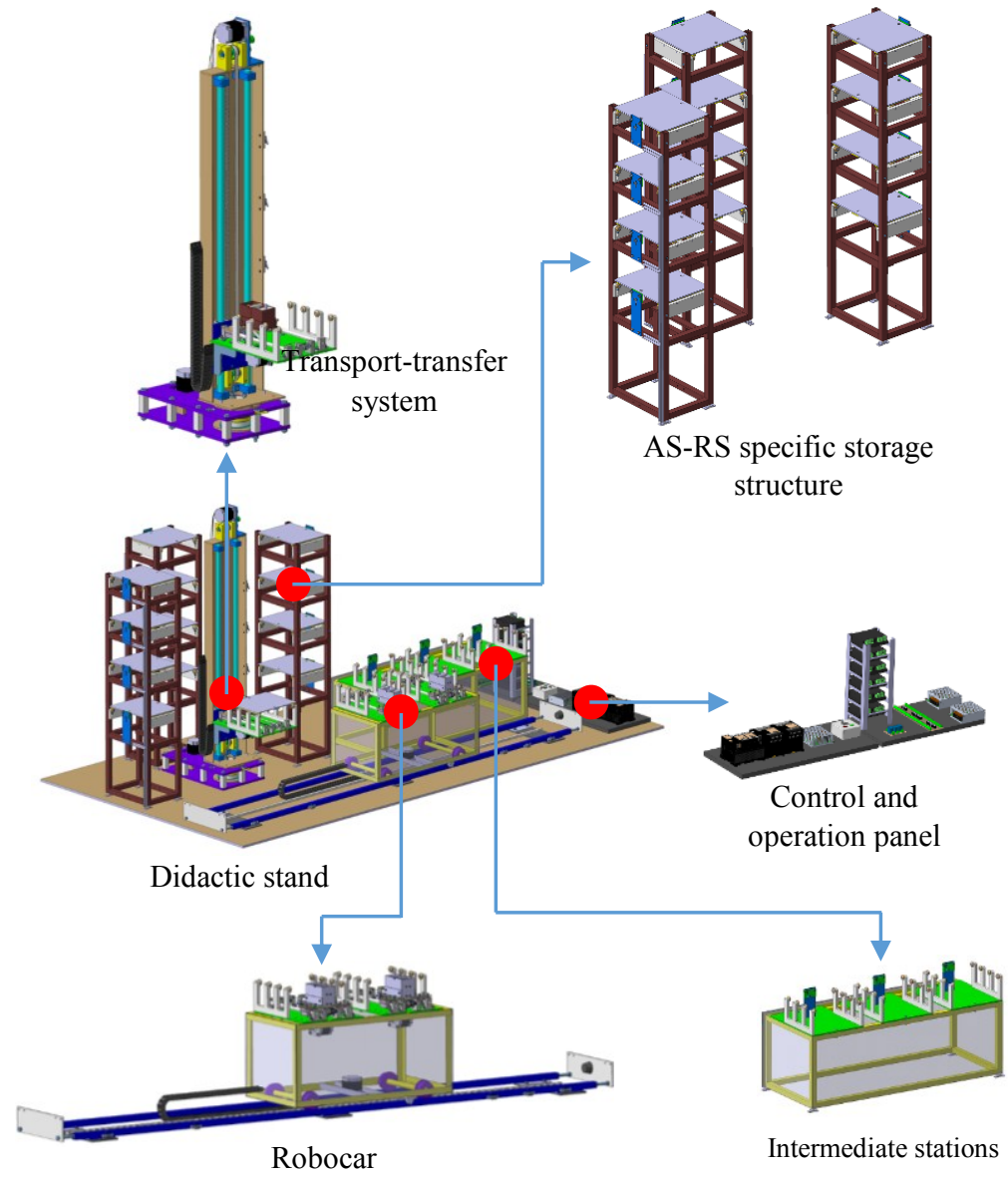

Fig. 1.The components of the didactic stand used for the experimental research[6] 
The control of the specific transport-transfer system AS-RS and of the robocar from the structure of the experimental stand is realized by means of 3 PLCs that communicate with each other, through input / output signals. In this sense, the PLCs use connections between the input and output ports, and the significance of the signals is established by the control program.

For testing stepper motors and translation and rotation movements, control programs made in CX Programmer and SCADA interfaces made in CX Supervisor were used. The language used is Ladder type and has predefined instructions that allow the control of stepper motors that operate the structures within the stand with acceleration and deceleration.

The movements performed on each $\mathrm{CN}$ axis specific to the presented systems, within the experimental stand, are represented in Figure 2.

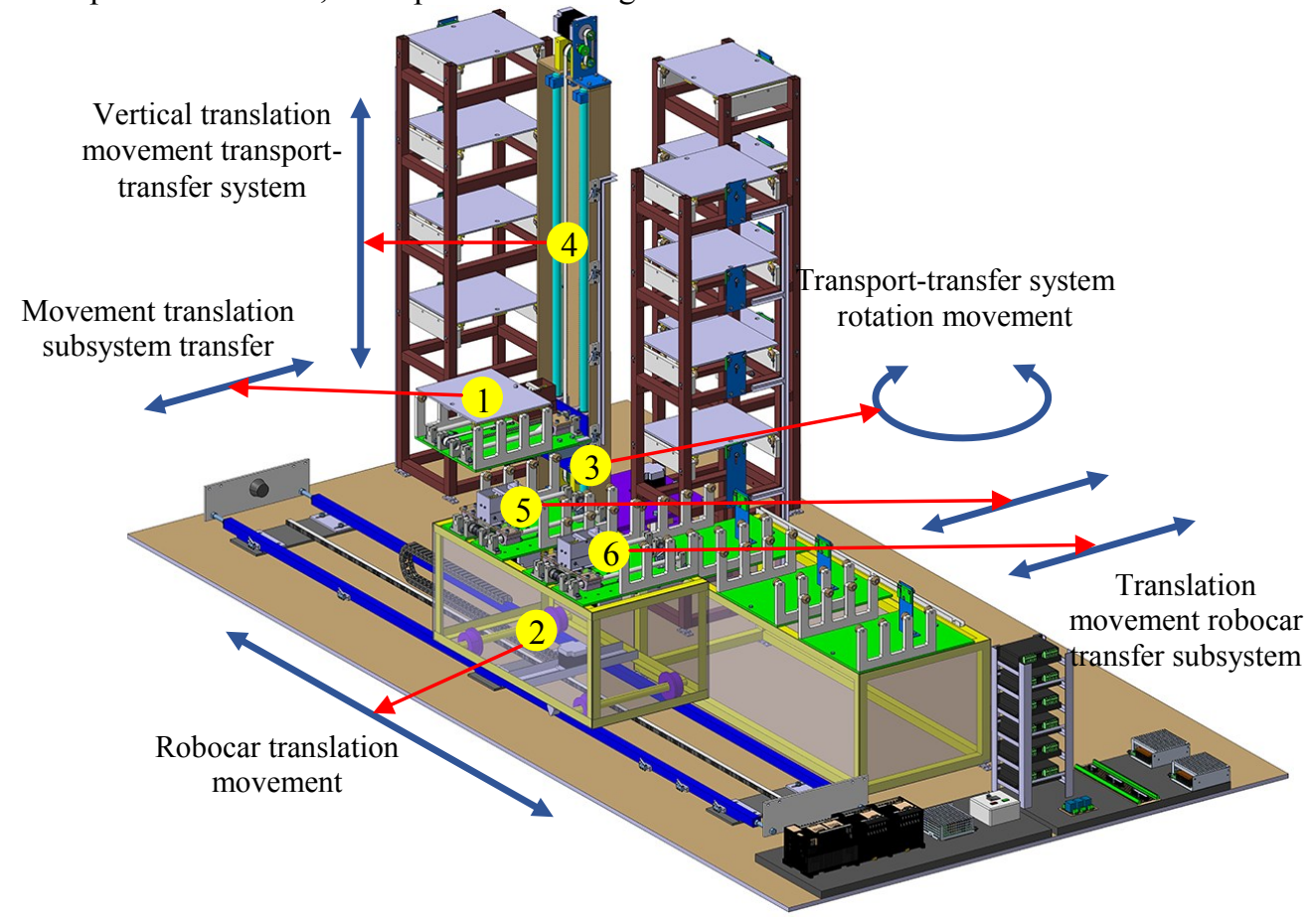

Fig. 2.NC axes driven by stepper motor[6]

\subsection{Experimental research to determine acceleration}

The application used for the command and control of the $\mathrm{CN}$ axes in order to perform the tests within the experimental researches, was made in the CX Supervisor work interface. Through it you can control the stepper motors in both directions.

The control interface allows the editing of the PLC memory. Thus it is possible to change in real time, the parameters of the stepper motor: acceleration, deceleration, frequency and number of pulses. Two operating modes can be selected for numerically controlled axes: (1) a single movement - press the button corresponding to the desired direction of rotation corresponding to the driven motor, and it performs a single rotation with the parameters entered by the user; (2) continuous movement - when operating the switch for continuous movement, the motor rotates in alternating directions using the parameters entered by the user. 
The stepper motor driving a $\mathrm{CN}$ axis that has an operating mode with acceleration and deceleration will determine a trapezoidal motion diagram. To draw this diagram it is necessary to determine four times shown in Figure 3:

- $\quad$ the moment of starting acceleration-t 1 ;

- $\quad$ the moment of the end of the acceleration period and the beginning of the movement period with constant speed $(\mathrm{a}=0)$ - $\mathrm{t} 2$;

- the momentof the end of the period of movement with constant speed and the beginning of the period of (deceleration)- $\mathrm{t} 3$;

- $\quad$ the moment of the end of the deceleration period, when the engine stops $(v=0)-t 4$;

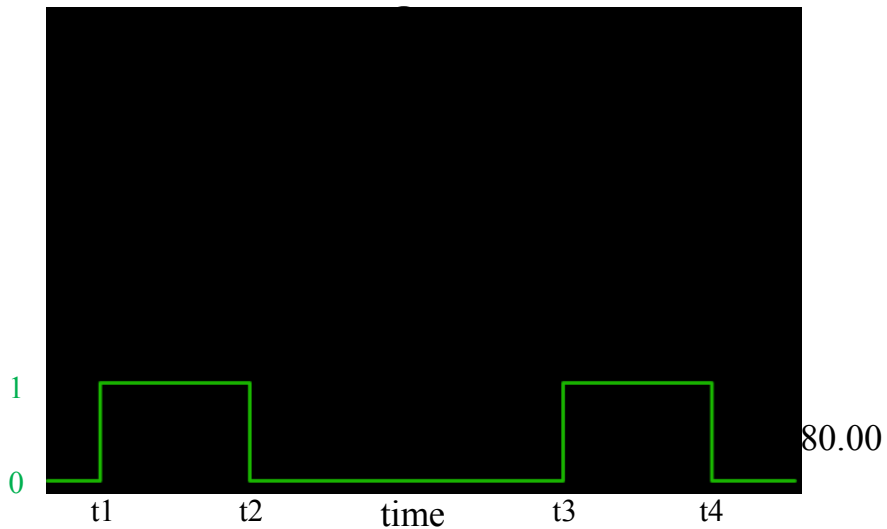

Fig. 3. Highlighting areas with accelerated / decelerated motion and constant speed on the motion diagram A280.00

Figure 3 highlights 3 distinct areas in relation to the 4 times mentioned, as follows:

- $\quad$ zone 1 - accelerated movement, from moment $\mathrm{t} 1$ - to moment $\mathrm{t} 2$;

- $\quad$ zone 2 - movement with constant speed, from moment $\mathrm{t} 2$ (moment of reaching the maximum frequency) to moment $\mathrm{t} 3$;

- zone 3 - decelerated movement, from the moment t3 (moment when the deceleration starts) to the moment 44 (the moment of stopping).

Using the A280.00 bit in the auxiliary memory area of the PLC, with the help of the SCADA interface the four times $\mathrm{t} 1-\mathrm{t} 4 \mathrm{can}$ be determined with an accuracy of $50 \mathrm{~ms}$.

Using times $\mathrm{t} 1 \mathrm{-t} 4$ and knowing the starting frequency $(0 \mathrm{~Hz})$ and the target frequency, different input-time diagrams can be drawn for the different input data presented in Table 1, which are shown in Figure 4.

Table 1. The values used for acceleration, deceleration and target speed

\begin{tabular}{|c|c|c|c|}
\hline Position & $\begin{array}{c}\text { Acceleration } \\
\text { (Hz/4ms) }\end{array}$ & $\begin{array}{c}\text { Deceleration } \\
\text { (Hz/4ms) }\end{array}$ & Frequency(Hz) \\
\hline 1 & 10 & 10 & 3000 \\
\hline 2 & 5 & 5 & 3000 \\
\hline 3 & 5 & 5 & 5000 \\
\hline 4 & 20 & 20 & 5000 \\
\hline 5 & 10 & 5 & 5000 \\
\hline
\end{tabular}




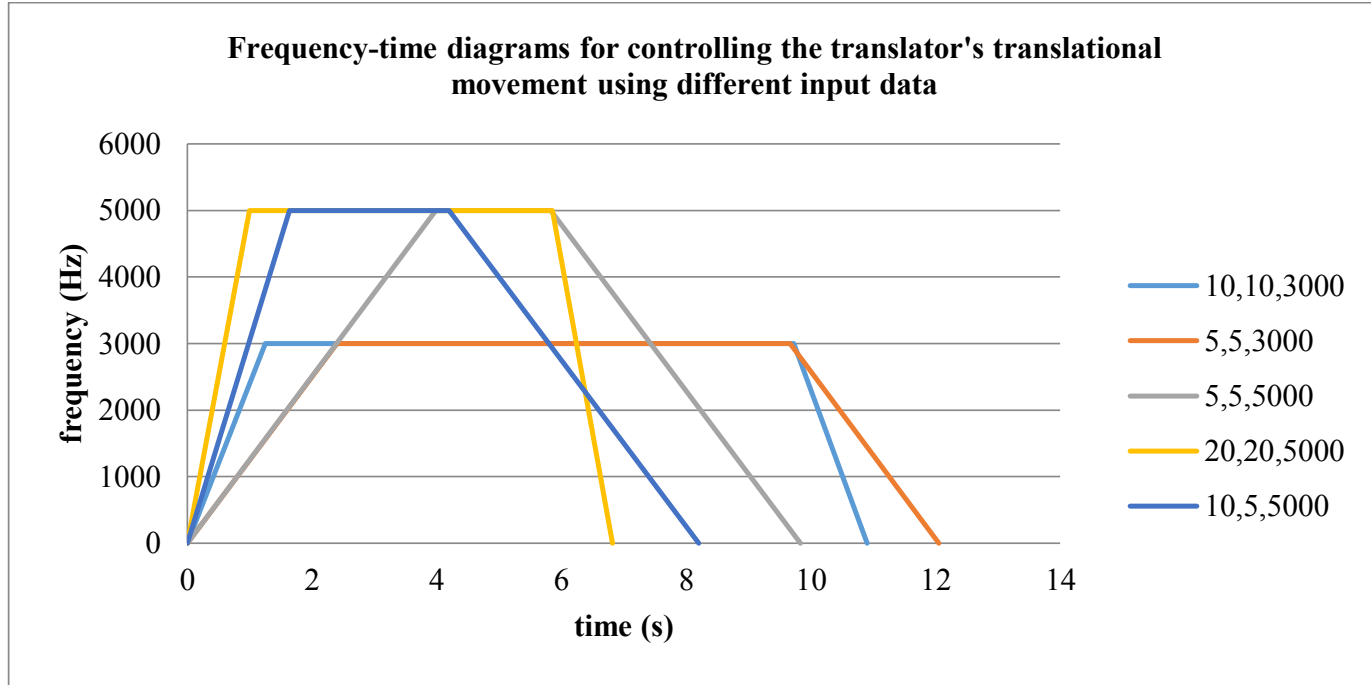

Fig. 4.Frequency-time diagram for controlling the robocar's translational movement

\subsection{Drawing motion diagrams using the SCADA application and the data provided by the programmable controllers}

Based on the frequency-time diagram, the motion diagram can be drawn, as the travel speed is directly proportional to the pulse generation frequency that controls the stepper motor. The speed is calculated based on the frequency, the resolution of the stepper motor driver and the motion transfer ratio, for each numerically controlled axis.

The displacement is calculated based on the number of pulses, the resolution of the stepper motor driver and the motion transfer ratio, for each numerically controlled axis.

Thus, a number of 10 tests were performed for each numerically actuated controlled axis, between the stroke limiters, with the parameters previously established, used as input data for determining the specific strokes and time intervals.

The average values obtained for the time intervals specific to each axis were centralized in Table 2.

Table 2.The values of the strokes and the average times on each $\mathrm{CN}$ axis

\begin{tabular}{|c|c|c|c|c|}
\hline Stepper motor & Rase & Dt2 (s) & Dt3 (s) & Dt4 (s) \\
\hline Robocar translation & $1364.0625 \mathrm{~mm}$ & 1.9928 & 5.9072 & 7.9202 \\
\hline AS-RS translation & $679.6875 \mathrm{~mm}$ & 0.5952 & 9.6729 & 10.2623 \\
\hline AS-RS rotation & $272.8125 \mathrm{O}$ & 0.4633 & 9.7883 & 10.1526 \\
\hline $\begin{array}{c}\text { Transfer system } \\
\text { translation }\end{array}$ & $210 \mathrm{~mm}$ & 1.1576 & 1.8402 & 3.1876 \\
\hline
\end{tabular}

Based on the data presented in the previous table, the movement diagrams for the translation of the robot (ground movement), the translation of the AS-RS specific transporttransfer system (vertical movement), the rotation of the AS-RS specific transport-transfer system (rotation) were drawn. at the base) and the translation specific to the transfer systems. The specific parameters of the transfer subsystems of the robot and the transport- 
transfer system within AS-RS were considered similar, as both the construction and the operation of these transfer systems are identical. Figures 5. a, 5.b, 5.c and 5.d show the motion diagrams for the aforementioned axes of motion.

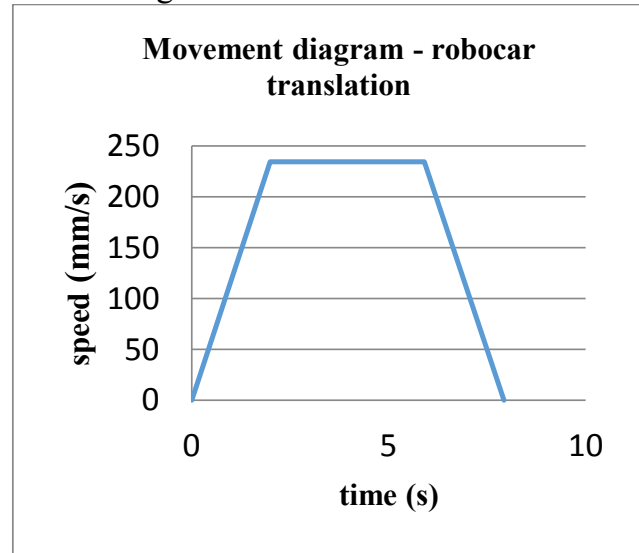

a) Movement diagram for the robocar's translation

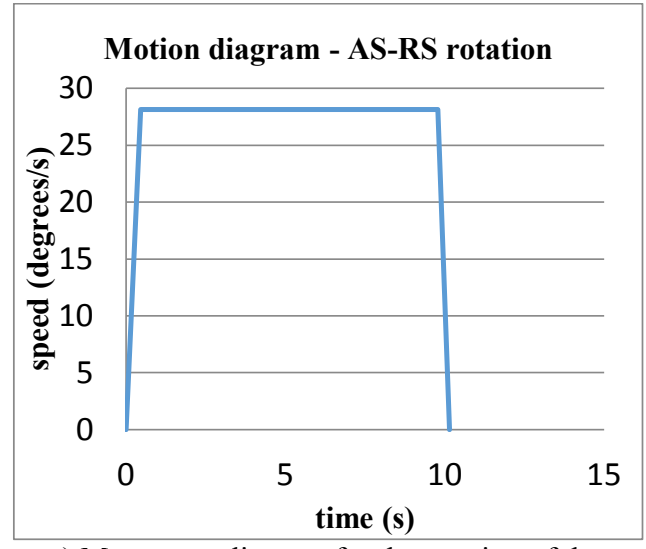

c) Movement diagram for the rotation of the transport-transfer system

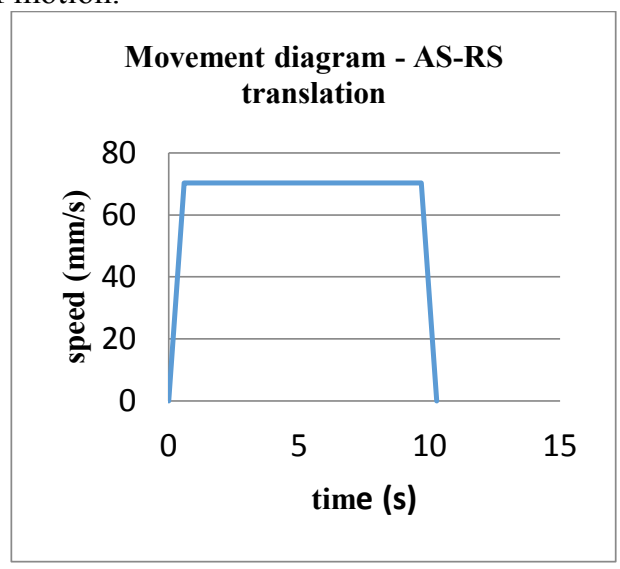

b) Movement diagram for the translation of the transport-transfer system

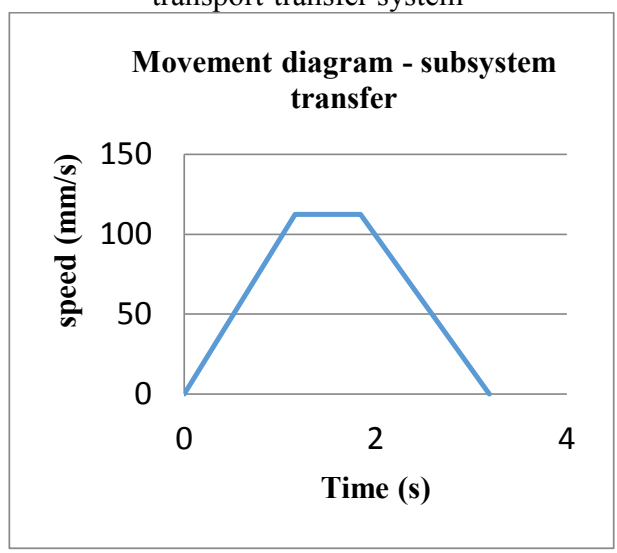

d)Movement diagram for pallet transfer system tranlation

Fig. 5.Specific movement diagrams to the $\mathrm{NC}$ axes of the structures within the experimental stand

\section{Case study - the complete operating cycle for storing a pallet}

In order to determine the total operating time specific to the movements of the $\mathrm{CN}$ axes of the systems within the educational stand, when loading a pallet, several tests were performed for different loading cycles specific to several stations within the AS-RS storage structure.

Thus, the movement diagrams for each $\mathrm{CN}$ axis of the specific transport-transfer system AS-RS for the maximum duration loading cycles were drawn. The diagrams presented in this paper were made for item no. 12 within the storage structure, which is the most difficult to access in terms of total storage time.

Figure 6 shows individually the movement diagram for the transfer subsystem when loading a blade in the storage structure specific to the AS-RS system. It can see the 4 movements corresponding to the following stages of a full loading cycle: extension of the transfer subsystem to take over the pallet (movement A), withdrawal of the transfer 
subsystem after taking over the pallet (movement B), extension of the transfer subsystem to deposit the pallet in the storage station (movement $\mathrm{C}$ ), withdrawing the transfer subsystem after depositing the pallet in the storage station (movement $\mathrm{D}$ ).

Also, in Figure 7 is presented the movement diagram for the vertical translation system, specific to the transport-transfer system when loading a pallet in the storage structure. You can also see in this figure the two positioning movements near the shock station after taking over the pallet, in order to store it, respectively to return to the initial position (entry-exit area AS-RS) after storing the pallet.

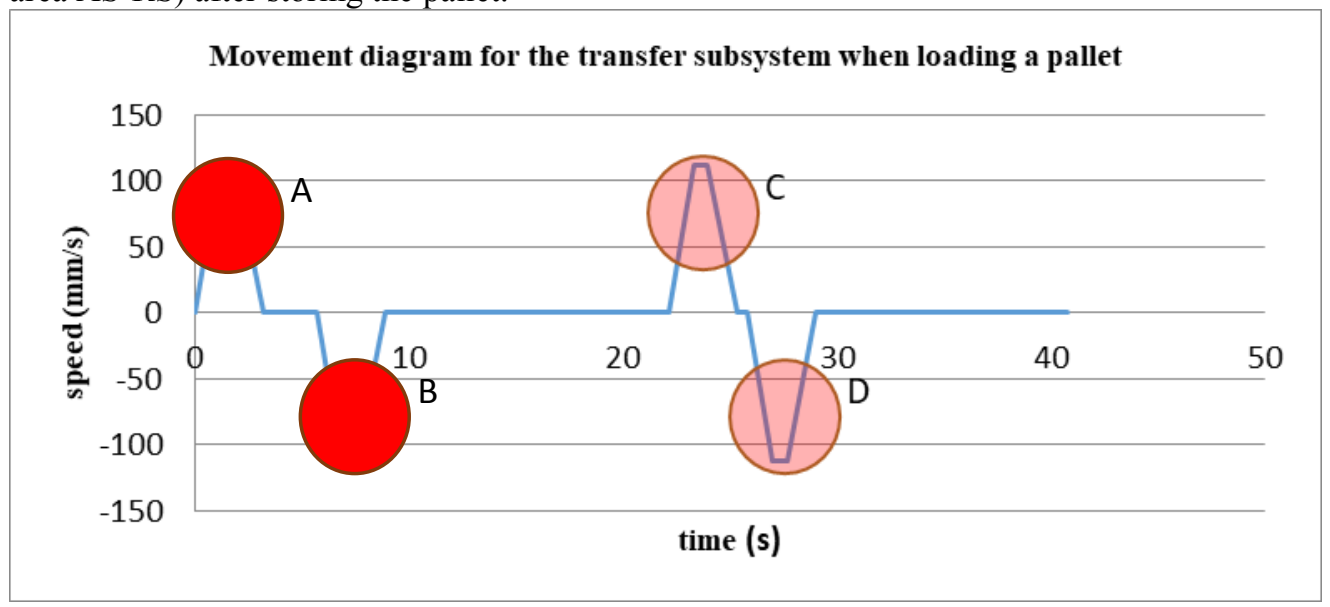

Fig. 6. Movement diagram for the transfer subsystem when loading a pallet

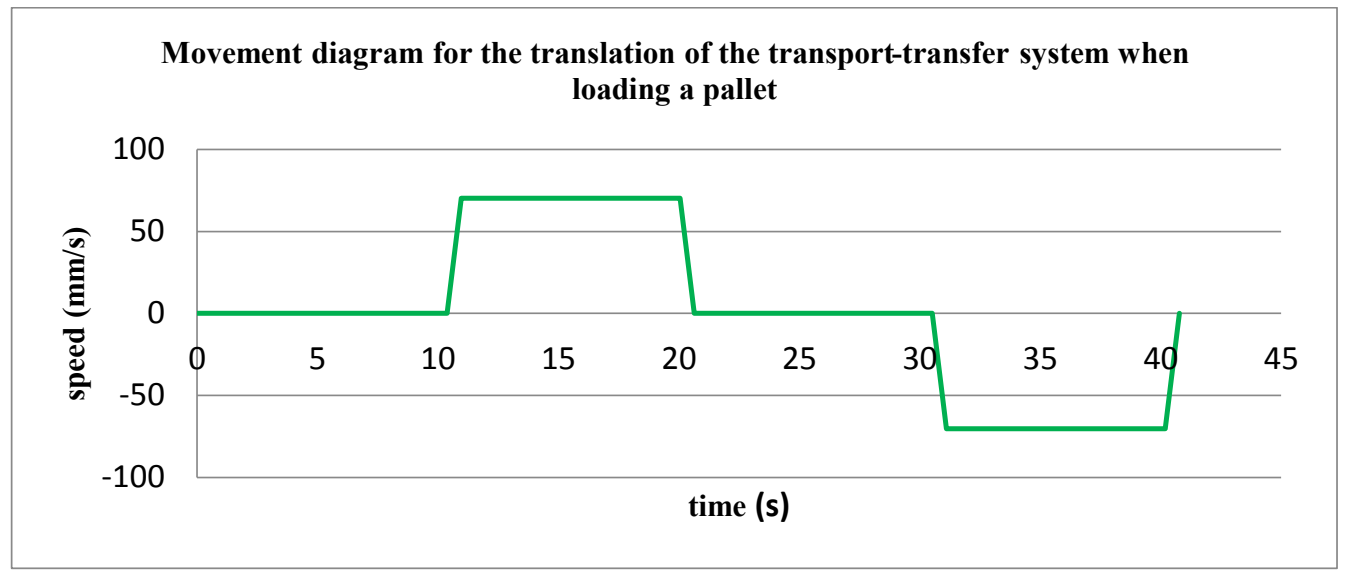

Fig. 7. Movement diagram for the translation of the transport-transfer system when loading a pallet

Similarly, Figure 8 shows the movement diagram for the rotation system at the base of the transport-transfer system when loading a pallet into the storage structure. Also in this figure are highlighted the two rotational movements for positioning next to the rack specific to the station where the pallet is to be stored, respectively to return to the initial position (AS-RS entry-exit area) after storing the pallet. 


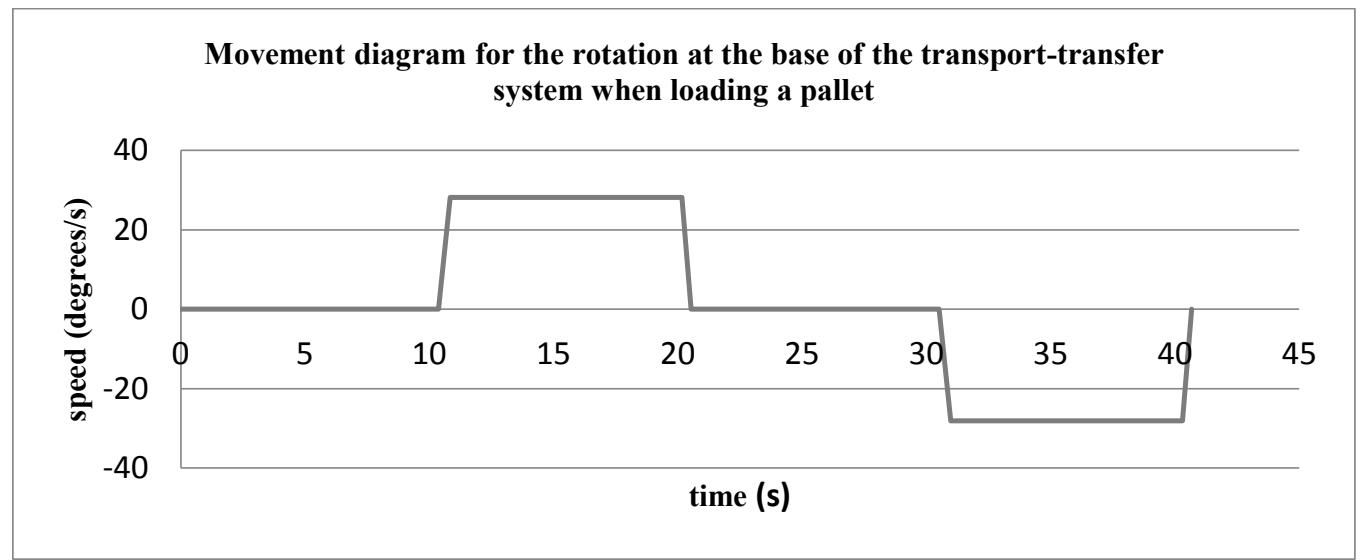

Fig. 8. Movement diagram for the rotation at the base of the transport-transfer system when loading a pallet

By overlapping the 3 diagrams corresponding to each numerically controlled axis of the transport-transfer system for an operating cycle, the movement diagram is obtained for a loading sequence of a pallet in the storage structure specific to the AS-RS system. This diagram is shown in Figure 9.

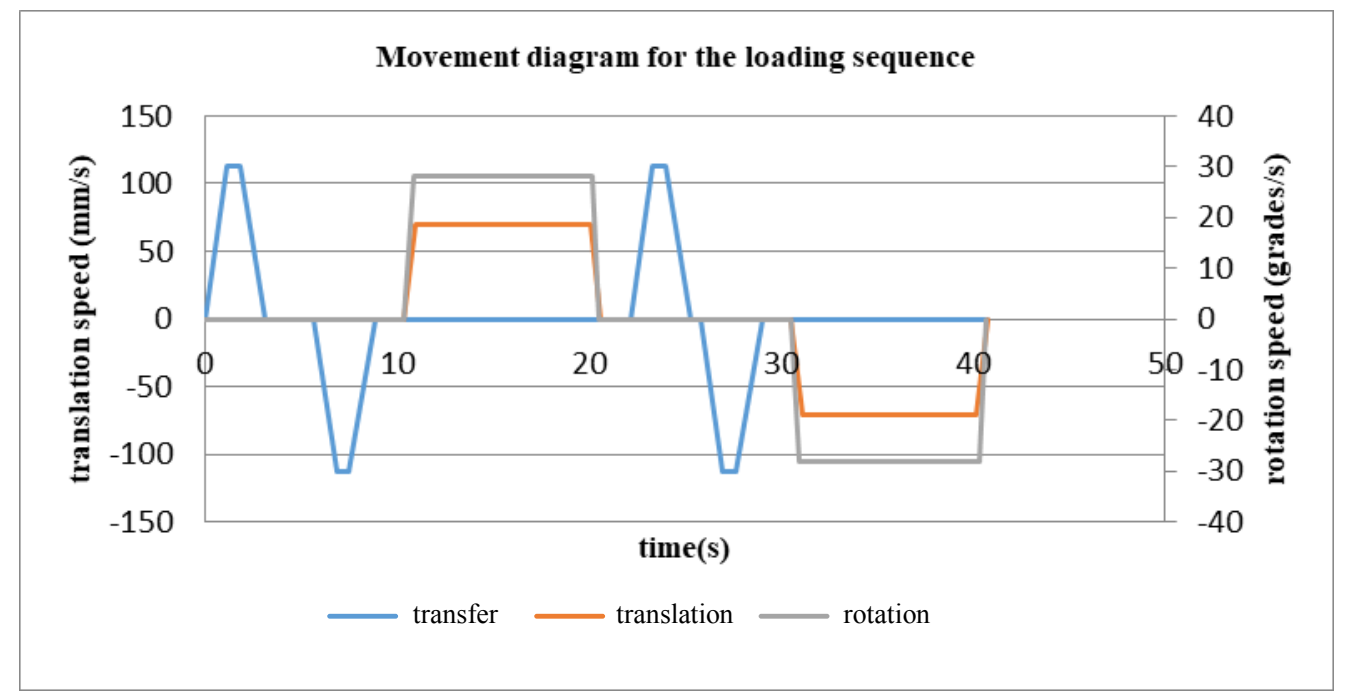

Fig. 9. Movement diagram of the $3 \mathrm{NC}$ axes specific to the transport-transfer system for a loading cycle

\section{Case study - the complete operating cycle for unloading a pallet from the storage structure}

For unloading a pallet from the storage structure, the movements performed on each $\mathrm{CN}$ axis specific to the transport-transfer system of the AS-RS, are performed in reverse order to the loading of a pallet. The time required for each movement of the $3 \mathrm{NC}$ axes is similar or identical for the two cases (loading and unloading a pallet), the storage station from which the unloading is done being the same. 
Thus, Figure 10 shows the movement diagram for the transfer subsystem when unloading a blade from the storage structure specific to the AS-RS system.

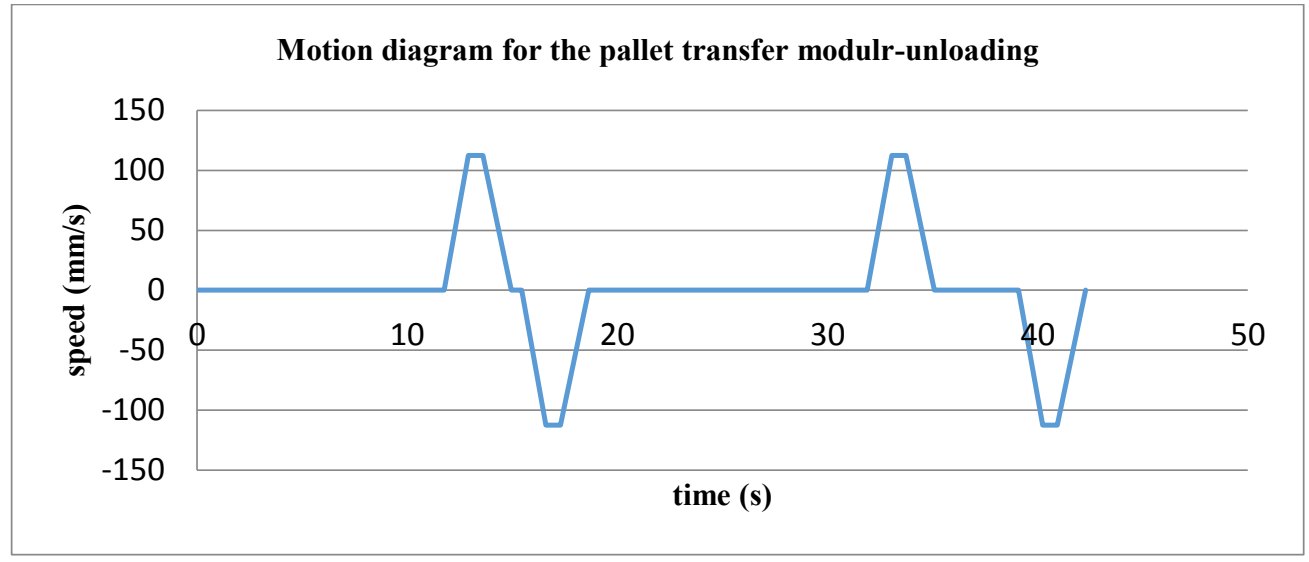

Fig. 10. Motion diagram for the transfer subsystem when unloading a pallet

Also, in Figure 11 is shown the movement diagram for the vertical translation system, and in Figure 12 is shown the movement diagram for the rotation module, when unloading the pallet from the storage structure specific to the AS-RS system.

After superimposing the 3 diagrams corresponding to each numerically controlled axis of the transport-transfer system for the operating cycle, the movement diagram is obtained for a sequence of unloading a pallet from the storage structure specific to the AS-RS system. This diagram is represented in figure 13 .

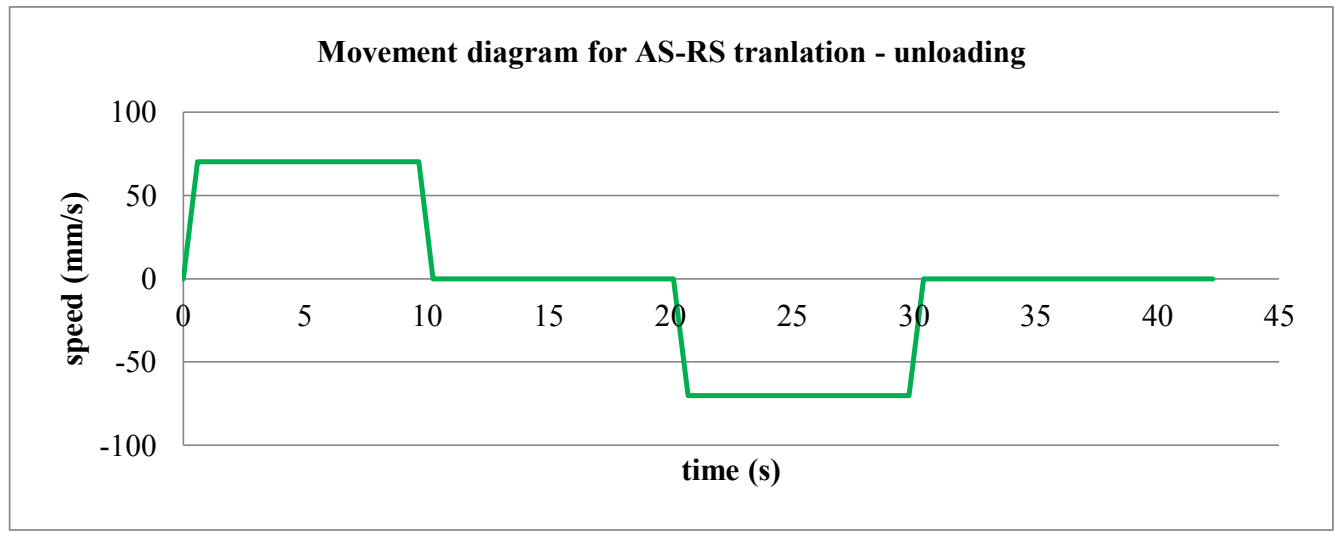

Fig. 11. Movement diagram for the translation of the transport-transfer system when unloading a pallet 


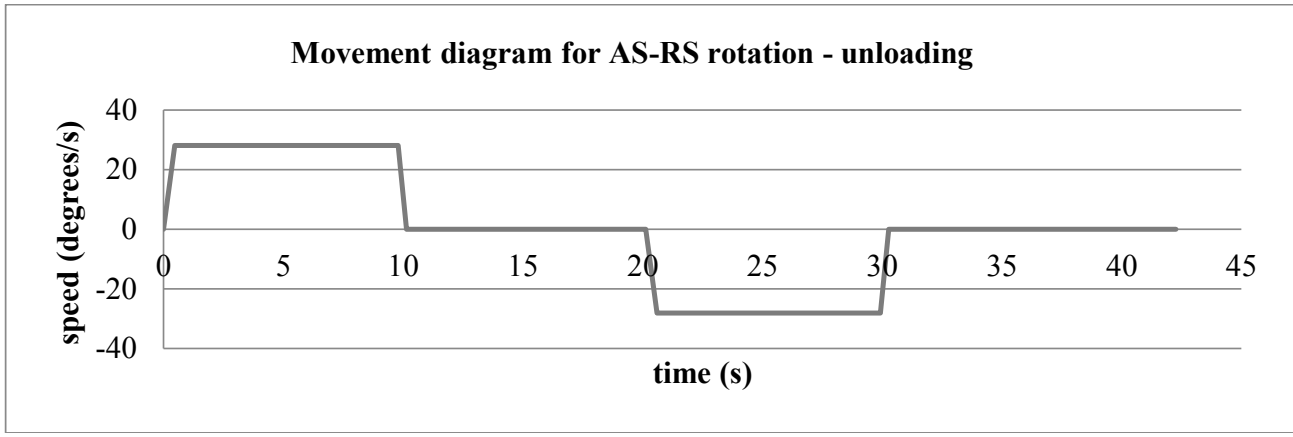

Fig. 12. Movement diagram for the rotation at the base of the transport-transfer system when unloading a pallet

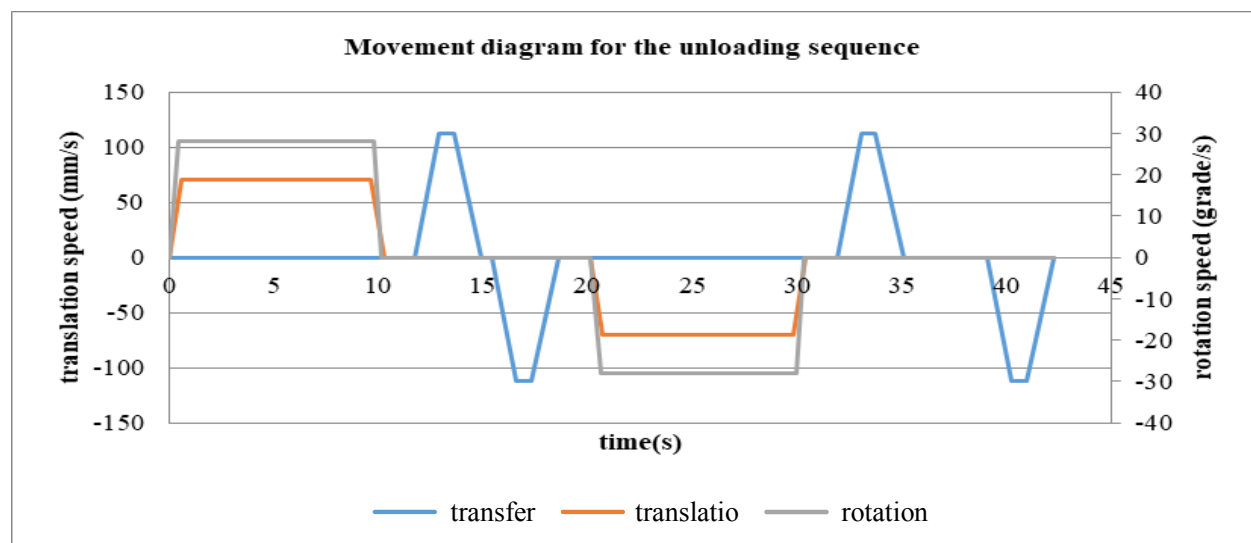

Fig. 13. Movement diagram of the transport-transfer system for the maximum duration unloading cycle

\section{Conclusions}

The operation of the technical systems is influenced by the way in which the motors for driving the $\mathrm{NC}$ axes from their structure are controlled.

Therefore, the speeds and accelerations used to control the $\mathrm{CN}$ axes within the educational stand used for experimental research, influence on the one hand the stability of the system as a whole, at the time of operation, and on the other hand the operating time for each axis. total service, but also the total service time.

Following the tests performed and the drawing of the movement diagrams for a complete loading-unloading cycle of a pallet, of maximum duration, the time intervals detailed in table 3 were obtained.

Table 3.The specific time for each operation, respectively the cumulative time for loading a pallet

\begin{tabular}{|c|c|c|}
\hline Operation & $\begin{array}{c}\text { Total time to load a pallet } \\
\text { (s) }\end{array}$ & $\begin{array}{c}\text { Total time to unload a } \\
\text { pallet (s) }\end{array}$ \\
\hline Standing next to the robocar & 10.38 & 11.88 \\
\hline Standing near the storage station & 9.88 & 9.88 \\
\hline
\end{tabular}




\begin{tabular}{|c|c|c|}
\hline Pallet transfer & 15.75 & 17.25 \\
\hline Vertical translation movement & 20.52 & 20.52 \\
\hline Rotation movement & 20.31 & 20.31 \\
\hline Unloading cycle time & 40.78 & 42.28 \\
\hline
\end{tabular}

It was thus possible to determine the total time required for the system to load a pallet in the storage station, respectively to unload a pallet from the storage station. The time differences for certain operations and implicitly between the duration of the complete loading and unloading cycles of a pallet, being introduced by the interaction of the specific transport-transfer system AS-RS with the robocar.

\section{References}

1. S. Ricci, V. Meacci, Electronics 7, 242 (2018)

2. S. Derammelaere, B. Vervisch, F.D. Belie, B. Vanwalleghem, J. Cottyn, P. Cox, G.V.D. Abeele, K. Stockman, L. Vandevelde, IEEE Ind. Appl. Mag. 20, 50-60 (2014)

3. M.Y. Stoychitch, ICAS 294, 1 (2017)

4. B. Wang, Q. Liu, L. Zhou, L. Bu, X. Li, J. Zhang, Electr. Mach. C., 22, 37-42, 52 (2018)

5. C.E. Cotet, C.L. Popa, G. Enciu, G.; A. Popescu, T. Dobrescu, IJSM, 15, 5 -15 (2016)

6. A. Popescu, Research on ERP applications for Small and Medium Enterprises effectiveness (2014) 